\title{
Steigern Antipsychotika Risiken für Lungenversagen?
}

COPD-Patienten unter Antipsychotika erkranken gehäuft an Lungenversagen. Das Risiko ist je nach Dosis um die Hälfte bis um das Vierfache erhöht. Ob das an den Arzneien oder schlicht an einem Behandlungsfehler liegt, ist aber unklar.

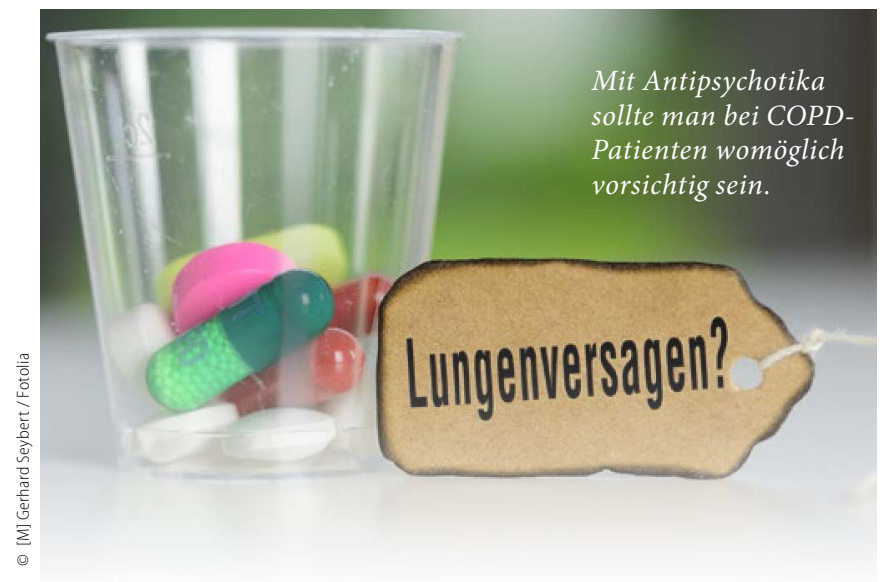

COPD-Patienten mit akutem Lungenversagen haben eine schlechte Prognose - jeder Zweite stirbt binnen eines Jahres. Es liegt daher nahe, mit Medikamenten bei COPD-Kranken vorsichtig zu sein, die sich ungünstig auf die Atmung auswirken. So gibt es Fallberichte von akutem Lungenversagen unmittelbar nach dem Therapiebeginn mit Antipsychotika, berichten Forscher um Dr. Meng-Ting Wang vom National Defense Medical Center in Taipeh (JAMA Psychiatry 2017, online 4. Januar).

Anhand einer großen taiwanesischen Datenbank haben die Ärzte geschaut, ob sich auch aus Patientenregistern ein erhöh- tes Risiko für Lungenversagen unter Antipsychotika ergibt. Dazu werteten sie Angaben zu praktisch allen Taiwanesen mit COPD aus, die zwischen 2000 und 2010 erstmalig aufgrund eines akuten Lungenversagens behandelt worden waren.

Patienten mit Lungenversagen in der Vorgeschichte waren ausgeschlossen worden, um sicherzugehen, dass eine eventuelle Antipsychotika-Behandlung den Atemproblemen vorausging. Das Team um Wang identifizierte nach diesen Kriterien 5.032 Patienten; 70 \% davon waren Männer, das Durchschnittsalter lag bei knapp 75 Jahren.

\section{Doppeltes Risiko nach Einnahme von Antipsychotika}

Bei diesen Patienten schauten sie nun in zwei unterschiedlichen Perioden, ob sie Antipsychotika bekommen hatten: in den 14 Tagen vor dem Lungenversagen und in den Tagen 88 bis 75 vor dem Ereignis. Diese Woche diente als Kontrollperiode. Medikamente - auch Depotneuroleptika - sollten nach spätestens zwei Monaten abgebaut sein und dann keine Auswirkungen auf die Atmung mehr haben. Den Antipsychotika-Gebrauch ermittelten die Ärzte anhand der ausgestellten Rezepte.

Ergebnis: In den beiden Wochen vor dem Lungenversagen nahmen 11,7\% der COPD-Kranken ein Neuroleptikum, aber nur $8,8 \%$ in der Kontrollwoche. Daraus errechneten die Forscher ein verdoppeltes Risiko für Lungenversagen nach der Einnahme von Antipsychotika.

Thomas Müller

\section{Grippewelle hat sich bundesweit ausgebreitet}

In diesem Jahr hat die Grippewelle besonders früh begonnen, berichtet die AG Influenza (AGI) am Robert Koch-Institut (RKI). So ist die Aktivität der akuten Atemwegserkrankungen (ARE) in der Woche bis zum 6. Januar (1. KW) stark gestiegen und liegt bundesweit im deutlich erhöhten Bereich. Besonders betroffen sind das Saarland und Rheinland-Pfalz sowie Sachsen, wo die ARE-Aktivität stark erhöht ist.

Ein großer Teil der Patienten mit Atemwegsinfekten hat Influenza: In Stichproben wurden bei etwa jedem sechsten ARE-Patienten Grippe-Viren nachgewiesen, fast alle vom Subtyp A (H3N2). In der 1. KW sind 2039 labordiagnostisch bestätigte Influenzafälle an das RKI übermittelt worden. Seit dem vergangenen Oktober (40. KW) waren es 6.709 bestätigte Fälle. Gut jeder vierte Betroffene wurde stationär behandelt. Nach Angaben der AGl gab es zudem fünf größere Ausbrüche in Kliniken mit mehr als fünf Betroffenen. Auch wurden in diesem Winter zwölf Todesfälle bei Influenza-Patienten registriert.

Laufende Untersuchungen in Finnland und Schweden deuten auf eine suboptimale Wirksamkeit des aktuellen Impfstoffs gegen die zirkulierenden
A(H3N2)-Viren hin. Die Impfwirksamkeit bei Menschen im Alter ab 65 Jahre liegt zurzeit nur bei 30\%. Daher sollte der frühzeitige Einsatz von antiviralen Arzneimitteln sowohl für geimpfte als auch nicht geimpfte Risikopatienten erwogen werden, betont die AGI. Wolfgang Geissel

In diesem Jahr breitet sich der Influenzavirus vom Subtyp $A$ (H3N2) besonders rasch aus.

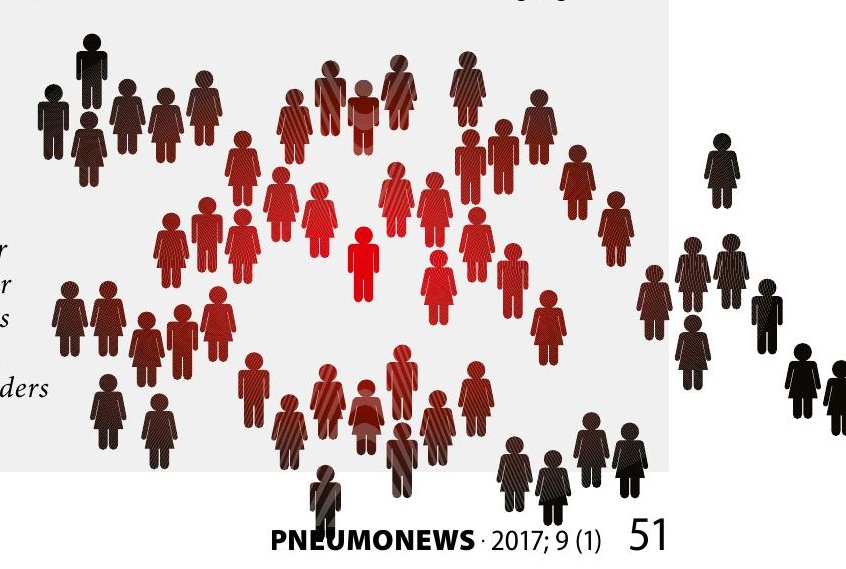

